\title{
Les visages de la tyrannie dans la trilogie romaine de Jacek Bocheński
}

\section{Aleksander Fiut}

Traducteur : Anna Saignes

\section{OpenEdition \\ Journals}

Édition électronique

URL : http://journals.openedition.org/recherchestravaux/505

DOI : 10.4000/recherchestravaux.505

ISSN : 1969-6434

Éditeur

UGA Éditions/Université Grenoble Alpes

Édition imprimée

Date de publication : 15 mai 2012

Pagination : 49-55

ISBN : 978-2-84310-227-1

ISSN : 0151-1874

Référence électronique

Aleksander Fiut, «Les visages de la tyrannie dans la trilogie romaine de Jacek Bocheński »,

Recherches \& Travaux [En ligne], 80 | 2012, mis en ligne le 15 novembre 2013, consulté le 07 septembre 2020. URL : http://journals.openedition.org/recherchestravaux/505 ; DOI : https://doi.org/10.4000/ recherchestravaux.505 
Aleksander Fiut

Université de Cracovie

(Traduit du polonais par Anna Saignes)

\section{Les visages de la tyrannie dans la trilogie romaine de Jacek Bocheński}

Parmi les paradoxes qui caractérisent la République populaire de Pologne - et aussi les autres pays du bloc de l'Est - il y a le rôle positif joué dans la culture par la censure. Sans nul doute, le crayon du censeur ravageait la littérature, précipitait d'un seul geste dans le néant des pans entiers de la réalité, condamnait à l'inexistence ou bien à une existence de façade certaines ouvres et certains auteurs, en particulier ceux de l'immigration, falsifiait l'Histoire et l'image de la réalité actuelle. D'un autre côté pourtant, l'effort permanent des écrivains, pour éviter l'ingérence de la censure et pour combattre l'autocensure, aboutissait en fin de compte à une grande adresse dans l'utilisation de la langue d'Ésope, à la mise au point de méthodes multiples et souvent subtiles pour transmettre des contenus politiquement condamnables voire interdits, à la multiplication de travestissements surprenants et de masques étonnants. Dans le jeu raffiné avec les prescriptions des censeurs, où primaient la ruse et l'inventivité, les paraboles historiques susceptibles d'une lecture politique occupaient une place importante. Le nombre de publications relevant de ce genre augmentait toujours durant les périodes charnières. Après octobre 1956, Jerzy Andrzejewski, dans son roman Les ténèbres recouvrent la terre, établit une analogie entre la période stalinienne et les temps de l'Inquisition espagnole. Andrzej Kijowski a tiré des conclusions de la révolte de 1968 dans l'essai Un soir de novembre, en apparence consacré à l'insurrection romantique. Les œuvres faisant référence à l'époque des partages de la Pologne constituaient un courant particulier qui soumettait à une analyse minutieuse les effets démoralisants de la privation prolongée de la liberté. 
Je pense par exemple à La Liberté de choisir de Mieczysław Jastrun, à La Fin du monde des chevau-légers de Marian Brandys, aux Horloges de Tomasz Lubieński, aux Visages et Lamentations de Władysław Terlecki. Ce ne sont là que quelques exemples, qui demanderaient à être étudiés à part.

Parmi toutes ces œuvres, la trilogie romaine de Jacek Bocheński occupe une place singulière et exceptionnelle. Les différents tomes ont été publiés à de longs intervalles : Le Divin Jules. Notes d'un antiquaire, en I96 $^{\mathrm{I}}$, Nason le poète en $1969^{2}$, César Tibère, en $2009^{3}$. Ce dernier tome a une genèse particulièrement intéressante : Bocheński a commencé à l'écrire en octobre 1970, s'en est détourné pour s'y remettre trente-cinq ans plus tard, en juin 2005, dans un contexte politique radicalement différent, et a terminé le tout en décembre 2008. Si toute la trilogie a vu le jour sur une période de près d'un demi-siècle, à première vue elle ne se rapporte que très indirectement à l'actualité politique. Bocheński met avant tout l'accent sur la modélisation de la tyrannie et de ses trois phases clés : la naissance, l'épanouissement et la décadence. Voilà qui invite à se demander jusqu'à quel point le récit de l'Histoire de l'Empire romain est une analyse camouflée des transformations intervenant dans l'Empire soviétique. Il est bien sûr possible de lire la trilogie comme une parabole, mais ce n'est pas là le but principal de l'auteur.

En y regardant de plus près, on peut constater que, dans son jeu avec la censure, Bocheński a mis en ouvre une combinaison de deux discours, que j'appellerais volontiers "un discours qui dissimule» et «un discours qui démasque». Le discours qui dissimule se caractérise par une grande cohérence et renvoie à l'Antiquité : il contient trois portraits d'empereurs romains, Jules César, Octave Auguste, et Tibère, élaborés en s'appuyant sur des chroniques, des lettres d'époque, et des études ultérieures d'historiens de l'Antiquité. Ce fonds documentaire est, de temps à autre, complété par les suppositions des narrateurs et des passages relevant de la pure fiction. Dans Le Divin Jules, beaucoup de citations et de commentaires renvoient aux chroniques de César, La Guerre des Gaules et La Guerre civile, à Cicéron ou à Atticus. Dans Nason le poète, il est largement fait référence aux ouvres d'Ovide. Dans César Tibère, on trouve une analyse fouillée des discours de Tacite, Suétone, Dion Casius et Valerius Maximus. La trilogie romaine constitue une pénétrante analyse psychologique des trois empereurs, sur fond d'un monde antique brossé avec élan et appuyé sur une connaissance rigoureuse de la réalité historique. Une telle lecture rend l'œuvre de Bocheński comparable à celles de Tadeusz Parnicki, Mika Waltari ou Umberto Eco.

I. J. Bocheński, Boski Juliusz, Zapiski antykwariusza, Varsovie, Czytelnik, 1961. (Désormais BJ)

2. Id., Nazo poeta, Varsovie, Czytelnik, I969. (Désormais NP)

3. Id., Tyberiusz Cezar, Varsovie, Świat Książki, 2009. (Désormais TC) 
Le lecteur attentif sera néanmoins frappé par des passages qui composent un tout autre discours, contenant des signaux nettement actualisants. Un rôle important est joué par les insertions d'expressions en langue de bois, du type : "ingérence dans les affaires intérieures de la Gaule» (BJ, p. 26), "étouffement des premières émeutes» ( $N P$, p. 186), "la mollesse des vieux réactionnaires" ( $B J$, p. 8I), "la relégation, c'est-à-dire l'éloignement» $(B J$, p. I86), etc. Lorsque, dans le récit du jugement de Dumnorix, l'accusé se voit reprocher le "sabotage économique", "la propagation du chaos et la démoralisation, la diversion, les agissements au profit de l'ennemi et la haute trahison» (BJ, p. 23), il est difficile de ne pas penser aux procès de Moscou. La terreur, la destruction des élites, la dévastation matérielle, «le crépuscule des idéaux anciens et la création de nouvelles notions, comme conséquence du chaos, de la corruption, des intrigues continuelles» (BJ, p. 37) qui accompagnent la conquête de la Gaule, rappellent inévitablement la soviétisation de l'Europe centrale. Jules César, qui, voulant devenir un dieu, «a créé un fanatisme désintéressé, lié au culte de sa personnalité» ( $B J$, p. 79), évoque nettement Staline. Lorsque Bocheński affirme qu' "Ovide n'a jamais été envahi par le doute sceptique, selon lequel l'Histoire peut avoir deux ordres, un ordre déterminé par le dieu régnant, et un autre par la résistance de fourmi que l'homme y oppose, et que l'écrivain, s'il est le porte-parole de l'Histoire, doit choisir entre ces deux ordres.» (NP, p. 9I), il est difficile de dire s'il est réellement question d'Antiquité ou du conflit entre le régime communiste et le milieu des écrivains après i968. D'autant plus que l'interrogatoire imaginaire d'Ovide est à l'image des interrogatoires du même type qui avaient lieu à l'époque en Pologne. De même, lorsque l'auteur parle de l'expulsion des Juifs de Rome en I9 après J.-C., comme de la première manifestation de l'"antisémitisme européen, préraciste bien évidemment, préchrétien", et commente ce fait en affirmant que «l'expulsion des Juifs» trahit «certaines caractéristiques universelles de la mentalité policière» ( $T C$, p. 195), il est difficile de résister à l'impression que l'exercice du pouvoir au nom de Tibère par le chef des services spéciaux, Sejan, est une allusion discrète à l'arrivée au pouvoir en Russie des membres du KGB.

Dans les passages de ce type, le discours qui dissimule semble céder la place au discours qui démasque. Ce dernier se caractérise certes par l'éparpillement, mais manifeste clairement sa présence à travers les références stylistiques et politiques à l'époque contemporaine. Plus précisément : entre les deux discours il y a toujours une tension, grâce à laquelle l'Histoire de la Rome antique acquiert une actualité inattendue, alors que les machinations du régime communiste composent une parabole sur les mécanismes éternels du pouvoir et la démoralisation des hommes qui y sont impliqués. 
Toutefois, ni la vérité universelle revêtue du costume antique, ni la démystification du système totalitaire ne constituent l'objectif dernier de Bocheński. Ce n'est pas par hasard si, dans Le Divin Jules, Bocheński a introduit le personnage de Nepos, un chroniqueur contemporain de César, qui a dissimulé des commentaires sur le règne de son maître dans des allusions à l'Histoire de la Grèce antique. Comme le dit Bocheński, «Cela devrait nous inciter à penser que le trait essentiel de l'allusion est son absence de référent précis, la relativité, la possibilité d'applications diverses, la généralité.» Il faut donc se garder d'«interpréter hâtivement les allusions et ne pas les associer aux choses auxquelles elles nous font penser. En effet, ces allusions concernent plutôt la nature du monde que les événements singuliers» ( $B J$, p. IOI). Même chose dans César Tibère, où un rôle semblable à celui de Nepos est joué par Fedrus, auteur de fables allégoriques sur les animaux, dans lesquelles «il a créé une philosophie de l'esclavage et a fixé les axiomes ironiques de celle-ci», avec le projet cependant de "montrer la vie seule et la nature des hommes» (TC, p. 56).

Se pose alors la question de savoir pourquoi Bocheński nous invite par moments à la lecture parabolique, alors qu'à d'autres moments il nous en dissuade, suggérant que le discours de l'œuvre doit être universel et intemporel. S'agit-il uniquement d'entraîner sur une fausse piste un censeur trop zélé? À quoi servent alors «l'absence de référent précis et la relativité de l'allusion "? Qu'exprime le désir d'accéder à la "nature du monde» et de montrer "la nature humaine»? Il vaut la peine de se souvenir que le discours qui dissimule dans César Tibère a dû être utilisé seulement dans la première partie du tome, intitulée "Prospekt 1970 ", alors que la deuxième partie, intitulée "Fantaisie 2005", a vu le jour dans la Pologne indépendante et n'était donc plus soumise à aucune restriction.

Il faut ici rappeler que le narrateur de Bocheński ne parle pas directement dans la trilogie, mais qu'il se manifeste à travers trois rôles significatifs : dans Le Divin Jules - comme le suggère le sous-titre de l'œuvre - il est un antiquaire; dans Nason le poète, un présentateur; dans César Tibère, il devient le guide d'une excursion. Grâce à ces transformations, dans le premier tome de la trilogie, l'Histoire devient une promenade à travers les textes; dans le deuxième, elle signifie une participation à un spectacle sur scène; et dans le troisième, elle nous transporte dans la sphère de la réalité virtuelle. L'auteur mène en même temps, consécutivement, une polémique avec la vision hégélienne et marxiste de l'Histoire, prend la défense de la souveraineté et de la liberté de l'écrivain menacées par la tyrannie, et fait enfin un diagnostic concernant la civilisation européenne $\mathrm{du} \mathrm{xx}^{\mathrm{e}}$ siècle. L'intervention de ces intermédiaires (l'antiquaire, le présentateur, le guide) met en cause la vision rationaliste, 
téléologique, misant sur le progrès de l'Histoire, et rappelle la conception de l'«Histoire sérielle» de Michel Foucault. Je ne prétends pas que Bocheński copie ou met à l'épreuve la justesse des vues du philosophe français. Les analogies sont toutefois frappantes et valent la peine d'être relevées.

Foucault dit ainsi :

L'historien [...] n'interprète plus le document pour saisir derrière lui une sorte de réalité sociale ou spirituelle qui se cacherait en lui; son travail consiste à manipuler et à traiter une série de documents homogènes concernant un objet déterminé et une époque déterminée, et ce sont les relations internes ou externes de ce corpus de documents qui constituent le résultat du travail de l'historien ${ }^{4}$.

Ainsi peuvent être dévoilés des événements imperceptibles pour les hommes des époques passées. "L'Histoire sérielle permet de faire apparaître en quelque sorte différentes couches d'événements (ibid.).» De cette façon, «l'Histoire, ce n'est donc pas une durée, c'est une multiplicité de durées qui s'enchevêtrent et ' 'enveloppent les unes les autres ${ }^{5} »$.

N'est-ce pas justement ainsi qu'agissent les intermédiaires de Bocheński ? Ils ne cessent de manipuler des documents, découvrant ainsi des choses dissimulées ou passées sous silence. L'antiquaire, par exemple, «saisit les débuts ou les fins des intrigues, mais il est effrayé par les silences inattendus, les histoires qui s'arrêtent en plein milieu» (BJ, p. 83), dont il doit raconter luimême la fin ou bien qu'il doit compléter. En revanche, le lecteur, grâce à cette manipulation habile des documents par les narrateurs, fait l'expérience d'une "multiplicité des durées», en même temps qu'il devient acteur des événements passés. Il peut, en tant que témoin muet, percer les intentions cachées des tyrans, suivre leurs machinations, intrigues et crimes, et en même temps ne jamais perdre de vue la réalité qui lui est familière, ni renoncer à sa conscience d'homme du $\mathrm{Xx}^{\mathrm{e}}$ siècle. Les fêlures dans la connaissance du passé permettent d'apprécier les hypothèses du narrateur au sujet du mystérieux exil d'Ovide, par exemple. Il se rend en même temps compte à quel point les événements qui font nettement basculer le cours de l'Histoire sont loin d'obéir à la dialectique, à quel point ils dérivent de la soif du pouvoir, de la peur de se compromettre ou du désir de survivre. Il suffit que quelqu'un, tel César, dénué de tout scrupule, et en même temps plein de charisme et doué d'une volonté de fer, éprouve le désir de devenir un dieu. Ou bien que quelqu'un d'autre, tel Octave, le porte-parole de l'ordre traditionnel, décide de dissimuler une vérité susceptible de provoquer un scandale familial. $\mathrm{Ou}$ bien que Tibère, le maitre de la dissimulation et le virtuose de l'hypocrisie,

4. M. Foucault, «Revenir à l'Histoire», dans M. Foucault, Dits et écrits II, I976-I988 [1972], Gallimard, coll. "Quarto», 200I, p. 277.

5. Ibid., p. 279. 
se sente menacé par l'ambitieux conseiller en qui il avait le plus confiance et l'anéantisse adroitement. C'est suffisant pour que le sang coule, pour qu'un grand poète soit condamné à l'exil, et pour que l'assassinat d'un chef des services secrets prépare la voie à la toute-puissance de ses successeurs.

Le monde fictionnel adopte dans le dernier tome de la trilogie romaine la forme d'un jeu électronique, dont le schéma reste certes fidèle aux faits historiques, mais qui gomme efficacement la frontière entre ce qui est réel et ce qui a été créé artificiellement, et qui, de plus, flatte les attentes et les goûts du grand public. Si dans Nason le poète, Les Métamorphoses d'Ovide nourrissent l'imagination poétique, dans César Tibère, la réalité a quelque chose de «cette fluidité, de cette fugacité [...] de ces spasmes du monde, de cette sensation que le sol se dérobe sous nos pieds, lorsque les concepts fondamentaux ne sont plus certains et que les genres littéraires sont en train de mourir» (TC, p. 5I). Le monde de ce roman se caractérise par une instabilité ontologique indépassable, qui se communique au narrateur, aux personnages et aux touristes qui participent à cette étrange excursion. La possibilité de s'incarner virtuellement dans les personnages historiques et de voyager dans le temps et l'espace ouvre des perspectives à couper le souffle. Mais en même temps Bocheński nous met en garde : cette possibilité peut également libérer les instincts les plus vils. La réalisation virtuelle de la "multiplicité des durées» conduit en effet au relativisme généralisé. Dans les médias où tout est autorisé et rien n'est interdit, on peut participer virtuellement aux séances du sénat ou être témoin des coups d'État. Mais on peut également entrer dans la conscience d'un bourreau en train de torturer une jeune fille, connaître le plaisir de violer et de tuer, s'adonner à des perversions sexuelles ou éprouver le sentiment de toute-puissance, comme l'empereur romain qui se proclame le maitre capricieux de la vie et de la mort.

Le discours de la trilogie romaine est particulièrement pessimiste. Il nous est plus facile de comprendre la Rome de la décadence, semble nous suggérer Bocheński, parce que nous sommes les témoins et les acteurs de la culture de l'épuisement. Les défenseurs des normes éthiques traditionnelles ont aujourd'hui l'air de radoteurs un peu grotesques et pitoyables, comme Caton autrefois. Dans Nason le poète, Bocheński croyait encore à la valeur de l'art qui dit la vérité et qui ne ploie pas sous les pressions extérieures, et apporte un soutien éternel. Dans César Tibère, il est dit qu'il n’y a plus de génies et que les spectacles deviennent de triviales orgies, mises en scène et imitant maladroitement des rituels religieux. De plus, le sort pitoyable de la république romaine, qui au moyen d'un reste de lois et d'institutions, dissimule, comme avec une feuille de figuier, la violence et l'arbitraire des tyrans, constitue un memento terrifiant pour chaque démocratie. Déjà César avait percé à 
jour les faiblesses de la république romaine, lorsqu'il avait dit : «la république sera démolie par celui qui permettra à ses institutions d'exister. Deux choses seulement sont nécessaires pour cela : l'armée et l'argent" (BJ, p. 76). En d'autres termes, la terreur et la corruption. Et pourtant le spectacle sanglant ne fait que commencer. Ses auteurs seront bientôt les successeurs de Tibère : Caligula, Néron et Domitien.

Bocheński semble donc proche d'une conception de l'Histoire comme phénomène caractérisé par la discontinuité, la multiplicité des couches et le pluralisme des événements. Mais il ne considère pas la reconstruction de l'Histoire, à l'instar de Foucault, comme une sphère exclusivement textuelle et axiologiquement neutre. La collision du discours qui dissimule et du discours qui démasque a dans son œuvre une dimension morale et moralisante. Ce qui est parfaitement compréhensible, étant donné les expériences historiques de l'auteur. Mais face au crime, à la bestialité et à l'humiliation les mots s'avèrent impuissants. C'est pourquoi Bocheński enferme sa relation aux victimes de la tyrannie dans des informations laconiques et des commentaires ironiques: "César écrivait de façon concise! Cette sincérité et ce laconisme! Ce grincement de phrases libres de toute fioriture et ces montagnes de cadavres, ces rivières pleines de noyés, ces villes incendiées, ces espaces dévastés, ces consciences achetées et les gloses du stratège!» (BJ, p. 37) L'ironie est l'expression de l'impuissance face au mal dans l'Histoire, mais elle devient un masque éloquent pour la compassion. 